\title{
Synchronization of Coupled Neutral-Type Neural Networks with Jumping-Mode-dependent Discrete and Unbounded Distributed Delays
}

\author{
Yurong Liu, Zidong Wang, Senior Member, IEEE, Jinling Liang and Xiaohui Liu
}

\begin{abstract}
In this paper, the synchronization problem is studied for an array of $N$ identical delayed neutral-type neural networks with Markovian jumping parameters. The coupled networks involve both the mode-dependent discrete time-delays and the mode-dependent unbounded distributed time-delays. All the network parameters including the coupling matrix are also dependent on the Markovian jumping mode. By introducing novel Lyapunov-Krasovskii functionals and using some analytical techniques, sufficient conditions are derived to guarantee that the coupled networks are asymptotically synchronized in mean square. The derived sufficient conditions are closely related with the discrete time-delays, distributed time-delays, mode transition probability and coupling structure of the networks. The obtained criteria are given in terms of matrix inequalities that can be solved efficiently by employing the semi-definite programme method. Numerical simulations are presented to further demonstrate the effectiveness of the proposed approach.
\end{abstract}

Index Terms-Synchronization; neutral-type neural networks; Markovian jumping systems; discrete time-delay; unbounded distributed time-delay; Kronecker product.

\section{INTRODUCTION}

$\mathbf{I}$ $\mathrm{N}$ the last decade, recurrent neural networks (RNNs) have drawn noticeable attention from many researchers working in a variety of areas such as signal and image processing, associative memories, combinatorial optimization and automatic control [1], [12], [18], [24], [30]. While traditional neural networks have been successfully applied in static data-based classification and prediction problems for various engineering systems, the dynamical behaviors of the RNNs have recently gained a lot of research interests due to their capabilities of using dynamical temporal behavior to process arbitrary sequences of inputs. Motivated from both the basic science and the technological practice, the study of synchronization problems among an array of neural networks has been an active topic of research in the past few years, see [13], [15], [20], [22], [23] for some recent publications. Note that the original notion of synchronization dates back to the $1980 \mathrm{~s}$

This work was supported in part by the Royal Society of the U.K., the National Natural Science Foundation of China under Grants 61074129, 61174136 and 61134009, and the Natural Science Foundation of Jiangsu Province of China under Grants BK2010313 and BK2011598.

Y. Liu is with the Department of Mathematics, Yangzhou University, Yangzhou 225002, P. R. China (email: liuyurong@gmail.com).

J. Liang is with the Department of Mathematics, Southeast University, Nanjing 210096, P. R. China.

Z. Wang and X. Liu are with the Department of Information Systems and Computing, Brunel University, Uxbridge, Middlesex, UB8 3PH, United Kingdom (email: Zidong.Wang@brunel.ac.uk). after the theory of deterministic chaos has been developed. Since then, the synchronization research has been extended to the case of more complex systems, for example, the largescale and complex networks of chaotic oscillators [14], [34], the coupled systems exhibiting spatio-temporal chaos and autowaves [28], [41], and the array of coupled neural networks with or without delays [7], [27], [37].

In practice, due to the finite speeds of the switching and transmitting signals, time delays exist in various RNNs [1], [16], [17]. It is well known that time delays may result in oscillatory behaviors or network instability (periodic oscillation and chaos). So far, most of the existing results related to the synchronization analysis for RNNs have been concerned with the discrete delay (point delay) case. Recently, the distributed delay has received an increasing research interest due to the presence of an amount of parallel pathways with a variety of axon sizes and lengths. Furthermore, as a combination of both discrete and distributed delays, the so-called mixed timedelays have gained much research attention and many relevant results have been reported in the literature, see e.g. [35], [38], [39] and the references therein. It should be pointed out that, rather than occurring in the system states (or outputs), timedelays can also appear in the derivatives of system states [4], [25], [26], [40]. This kind of time-delays is referred to as the neutral time-delays that can find a variety of applications in practice such as chemical reactors, transmission lines, partial element equivalent circuits in VLSI systems, and LotkaVolterra systems [8]. Because of possible presence of neutral delays in implementing RNNs in VLSI circuits, the RNNs with neutral terms have stirred some attention in the past few years, see e.g. [8], [9], [19].

During the course of implementation, the RNNs often encounter the information latching problems [5], that is, the network states have finite representations (also called clusters, patterns, or modes) where the switching among the finite states is sometimes governed by a Markovian chain. Such kind of random mode switches may result from abrupt phenomena such as stochastic failures and repairs of the network components, changes in the interconnections of network nodes, or sudden environment switching. As such, the so-called Markovian jumping recurrent neural networks (MJRNNs) have attracted a great deal of research interest [31], [36], [42] in the past decade. For example, in [36], the exponential stability problem has been first addressed for a class of delayed recurrent neural networks with Markovian jumping parameters. In [42], the problem of exponential stability has 
been investigated for a class of stochastic neural networks with both Markovian jump parameters and mixed time delays. In [31], a noise-induced stabilization method has been proposed for RNNs with mixed time-varying delays and Markovian switching parameters. In [38], the passivity analysis has been conducted for discrete-time stochastic neural networks with both Markovian jumping parameters and mixed time delays.

Summarizing the discussion made so far, the RNNs often exhibit the phenomena of signal transmission delays and possess Markovian mode jumping behavior, where the delays could be of discrete, distributed and neutral types. As such, it should be of both theoretical and practical significance to consider the synchronization problem of such RNNs. Unfortunately, the synchronization issue for Markovian jumping neutral-type neural networks with mode-dependent mixed time-delays has received very little research effort due primarily to the mathematical complexity. It is, therefore, the motivation of our current investigation to shorten such a gap by launching a study on the synchronization problem for Markovian jumping neural networks of neutral type where all discrete, distributed and neutral delays are mode-dependent and the distributed delays are allowed to be unbounded. It is noticeable that, in two recent papers [2], [3], the passivity and stability analysis problems have been addressed for neural networks of neutral type with Markovian jumping parameters and time delays, where the time-delays are not mode-dependant.

In this paper, we are concerned with the synchronization problem for a new class of continuous-time neural networks of neutral-type with Markovian jumping parameters as well as mode-dependent mixed time-delays. Note that the mixed time-delays comprise both the discrete and distributed delays that are all dependent on the Markovian jumping mode. The main contributions of this paper can be highlighted as follows: 1) some novel analysis techniques are developed to tackle the mathematical difficulty resulting from the presence of the mode-dependent neutral delays; 2) a new Lyapunov functional is proposed to reflect the Markovian jumps of the delay bounds; and 3) a unified framework is established to handle the Markovian jumping parameters, neutral terms and mixed time-delays. We derive sufficient conditions to guarantee that the coupled networks are asymptotically synchronized in mean square. Note that the derived sufficient conditions are expressed by means of the system parameters, discrete timedelays, distributed time-delays, mode transition probability and coupling structure of the array of neural networks. Such conditions are in the form of LMIs, which could be easily checked by utilizing the recently developed interior-point methods available in Matlab toolbox, and no turning of parameters will be needed. Numerical simulations are presented to further demonstrate the effectiveness of the proposed approach.

Notations: The notations are quite standard. Throughout this paper, $\mathbb{R}^{n}$ and $\mathbb{R}^{n \times m}$ denote, respectively, the $n$ dimensional Euclidean space and the set of all $n \times m$ real matrices. The superscript " $T$ " denotes matrix transposition and the notation $X \geq Y$ (respectively, $X>Y$ ) where $X$ and $Y$ are symmetric matrices, means that $X-Y$ is positive semidefinite (respectively, positive definite). $I_{n}$ is the $n \times n$ identity matrix. $|\cdot|$ is the Euclidean norm in $\mathbb{R}^{n}$.
If $A$ is a matrix, denote by $\|A\|$ its operator norm, i.e., $\|A\|=\sup \{|A x|:|x|=1\}=\sqrt{\lambda_{\max }\left(A^{T} A\right)}$ where $\lambda_{\max }(\cdot)$ (respectively, $\left.\lambda_{\min }(\cdot)\right)$ means the largest (respectively, smallest) eigenvalue of $A$. The Kronecker product of an $n \times m$ matrix $X$ and a $p \times q$ matrix $Y$ is defined by an $n p \times m q$ matrix $X \otimes Y$ as follows

$$
X \otimes Y=\left[\begin{array}{ccc}
x_{11} Y & \cdots & x_{1 m} Y \\
\vdots & & \vdots \\
x_{n 1} Y & \cdots & x_{n m} Y
\end{array}\right] .
$$

The asterisk $*$ in a matrix is used to denote term that is induced by symmetry. $\mathbb{E}[x]$ and $\mathbb{E}[x \mid y]$ will, respectively, mean the expectation of $x$ and the expectation of $x$ conditional on $y$. Matrices, if their dimensions are not explicitly stated, are assumed to be compatible for algebraic operations.

\section{PRoblem Formulation}

Let $r(t)(t \geq 0)$ be a right-continuous Markov chain on a probability space taking values in a finite state space $\mathscr{N}=$ $\left\{1,2, \ldots, n_{0}\right\}$ with generator $\Pi=\left\{\pi_{i j}\right\}$ given by

$P\{r(t+\Delta)=j \mid r(t)=i\}= \begin{cases}\pi_{i j} \Delta+o(\Delta), & \text { if } i \neq j, \\ 1+\pi_{i j} \Delta+o(\Delta), & \text { if } i=j .\end{cases}$

Here $\Delta>0$, and $\pi_{i j} \geq 0$ is the transition rate from $i$ to $j$ if $j \neq i$ while

$$
\pi_{i i}=-\sum_{j \neq i} \pi_{i j}
$$

For a given array of $N$ identical neutral-type neural networks, we assume that each single neural network consists of $n$ neurons and the dynamics of $k$ th neutral-type neural network is governed by

$$
\begin{aligned}
\dot{x}_{k}(t)=E & (r(t)) \dot{x}_{k}\left(t-\tau_{1, r(t)}\right)-A(r(t)) x_{k}(t) \\
& +B(r(t)) f\left(x_{k}(t)\right)+C(r(t)) g\left(x_{k}\left(t-\tau_{2, r(t)}\right)\right) \\
& +D(r(t)) \int_{-\infty}^{t-\tau_{3, r(t)}} \varphi(t-s) h\left(x_{k}(s)\right) d s+u(t),
\end{aligned}
$$

where $x_{k}(t)=\left[x_{k 1}(t), x_{k 2}(t), \cdots, x_{k n}(t)\right]^{T}$ is the state vector of the $k$ th delayed neural network; $A(r(t))=$ $\operatorname{diag}\left\{a_{1}(r(t)), a_{2}(r(t)), \ldots, a_{n}(r(t))\right\}>0$ is a diagonal matrix with $a_{j}$ representing the rate with which the $j$ th neuron will reset its potential to the resting state in isolation; $B(r(t))=\left(b_{i j}(r(t))\right)_{n \times n}, C(r(t))=\left(c_{i j}(r(t))\right)_{n \times n}$, $D(r(t))=\left(d_{i j}(r(t))\right)_{n \times n}$ and $E(r(t))=\left(e_{i j}(r(t))\right)_{n \times n}$ denote connection weight matrices of the neurons; $u(t)=$ $\left[u_{1}(t), \ldots, u_{n}(t)\right]^{T}$ is the input vector function; and $f(\cdot)=$ $\left(f_{1}(\cdot), f_{2}(\cdot), \ldots, f_{n}(\cdot)\right)^{T}, \quad g(\cdot)=\left(g_{1}(\cdot), g_{2}(\cdot), \ldots, g_{n}(\cdot)\right)^{T}$, $h(\cdot)=\left(h_{1}(\cdot), h_{2}(\cdot), \ldots, h_{n}(\cdot)\right)^{T}$ denote the activation function vectors; $\tau_{1, r(t)}$ and $\tau_{2, r(t)}$ denote the mode-dependent discrete time delays while $\tau_{3, r(t)}$ characterizes the mode-dependent upper bound of the distributed time-delay.

Consider the following linearly coupled dynamical system comprising the above $N$ identical neutral-type neural net- 
works:

$$
\begin{aligned}
\dot{x}_{k}(t)=\dot{x}_{k}( & \left.t-\tau_{1, r(t)}\right)-A(r(t)) x_{k}(t)+B(r(t)) f\left(x_{k}(t)\right) \\
& +C(r(t)) g\left(x_{k}\left(t-\tau_{2, r(t)}\right)\right) \\
& +D(r(t)) \int_{-\infty}^{t-\tau_{3, r(t)}} \varphi(t-s) h\left(x_{k}(s)\right) d s+u(t) \\
& +\sum_{j=1}^{N} w_{k j}(r(t)) \Gamma(r(t)) x_{j}(t), k=1,2, \ldots, N, \quad(2)
\end{aligned}
$$

where $\Gamma(r(t))=\operatorname{diag}\left(\gamma_{1}(r(t)), \gamma_{2}(r(t)), \ldots, \gamma_{n}(r(t))\right) \geq 0$ is a diagonal matrix linking the $j$ th state variable of each neural network if $\gamma_{j}(r(t)) \neq 0 ; W=\left(w_{i j}(r(t))\right) \in \mathbb{R}^{N \times N}$ is the coupling configuration matrix of the system with $w_{i j}(r(t)) \geq$ $0(i \neq j)$ but not all zero.

Remark 1: In the array of coupled neural networks (2), the distributed delay $\int_{-\infty}^{t-\tau_{3, r(t)}} \varphi(t-s) h\left(x_{k}(s)\right) d s$ is included with the upper bound dependent on the Markov chain. Note that the time-delay $s$ can vary from $-\infty$ to $t-\tau_{3, r(t)}$ in a distributed way. As such, the unboundedness and the mode-dependence of such a distributed time-delay would have a great impact on the stability analysis on the overall coupled system. For the practical applications of such unbounded distributed delays, we refer the authors to [10], [11], [21]. It is worth mentioning that the finite distributed delays, which are another type of distributed delays whose lower and upper bounds are both limited, have been intensively investigated in [35], [38], [39].

Throughout this paper, we make the following assumptions.

Assumption 1: The coupling configuration matrix $W(r(t))=\left(w_{i j}(r(t))\right)$ is symmetric (i.e., $W(r(t))=$ $\left.W^{T}(r(t))\right)$ and satisfies

$$
\sum_{j=1}^{N} w_{i j}=\sum_{j=1}^{N} w_{j i}=0, i=1,2, \ldots, N .
$$

Assumption 2: As in [21], for $j \in\{1,2, \ldots, n\}, \forall s_{1}, s_{2} \in$ $\mathbb{R}, s_{1} \neq s_{2}$, the neuron activation functions satisfy

$$
\begin{aligned}
& l_{j}^{-} \leq \frac{f_{j}\left(s_{1}\right)-f_{j}\left(s_{2}\right)}{s_{1}-s_{2}} \leq l_{j}^{+}, \\
& \sigma_{j}^{-} \leq \frac{g_{j}\left(s_{1}\right)-g_{j}\left(s_{2}\right)}{s_{1}-s_{2}} \leq \sigma_{j}^{+}, \\
& v_{j}^{-} \leq \frac{h_{j}\left(s_{1}\right)-h_{j}\left(s_{2}\right)}{s_{1}-s_{2}} \leq v_{j}^{+},
\end{aligned}
$$

where $l_{j}^{-}, l_{j}^{+}, \sigma_{j}^{-}, \sigma_{j}^{+}, v_{j}^{-}, v_{j}^{+}$are some constants.

Remark 2: As discussed in [21], the constants $l_{j}^{-}, l_{j}^{+}, \sigma_{j}^{-}$, $\sigma_{j}^{+}, v_{j}^{-}, v_{j}^{+}$in Assumption 2 are allowed to be positive, negative or zero. Hence, the resulting activation functions could be non-monotonic, and more general than the usual sigmoid functions. In addition, when using Lyapunov stability theory to analyze the stability, such a description is particularly suitable since it quantifies the lower and upper bounds of the activation functions that offer the possibility of reducing the induced conservatism.

Assumption 3: The delay kernel $\varphi(\cdot):[0,+\infty) \rightarrow[0,+\infty)$ is continuous and integrable, and also satisfies

$$
\int_{0}^{+\infty} \varphi(s) d s<+\infty, \quad \int_{0}^{+\infty} s \varphi(s) d s<+\infty .
$$

Let

$$
\begin{aligned}
x(t) & =\left(x_{1}^{T}(t), x_{2}^{T}(t), \ldots, x_{N}^{T}(t)\right)^{T}, \\
\mathbf{f}(x(t)) & =\left(f^{T}\left(x_{1}(t)\right), f^{T}\left(x_{2}(t)\right), \ldots, f^{T}\left(x_{N}(t)\right)\right)^{T}, \\
\mathbf{g}(x(t)) & =\left(g^{T}\left(x_{1}(t)\right), g^{T}\left(x_{2}(t)\right), \ldots, g^{T}\left(x_{N}(t)\right)\right)^{T}, \\
\mathbf{h}(x(t)) & =\left(h^{T}\left(x_{1}(t)\right), h^{T}\left(x_{2}(t)\right), \ldots, h^{T}\left(x_{N}(t)\right)\right)^{T}, \\
\mathbf{u}(t) & =\left(u^{T}(t), u^{T}(t), \ldots, u^{T}(t)\right)^{T} .
\end{aligned}
$$

With the above symbols and the Kronecker product of matrices, we rewrite the system (2) in the following compact form:

$$
\begin{aligned}
\dot{x}(t)=( & \left.I_{N} \otimes E(r(t))\right) \dot{x}\left(t-\tau_{1, r(t)}\right)-\left(I_{N} \otimes A(r(t))\right) x(t) \\
& +\left(I_{N} \otimes B(r(t))\right) \mathbf{f}(x(t))+\left(I_{N} \otimes C(r(t))\right) \\
& \times \mathbf{g}\left(x\left(t-\tau_{2, i}\right)\right)+\left(I_{N} \otimes D(r(t))\right) \int_{-\infty}^{t-\tau_{3, r(t)}} \varphi(t-s) \mathbf{h}(x(s)) d s \\
& +\mathbf{u}(t)+W(r(t)) \otimes \Gamma(r(t)) x(t) .
\end{aligned}
$$

Definition 1: The coupled system (2) or (8) is said to be globally asymptotically synchronized in mean square if

$$
\lim _{t \rightarrow \infty} \mathbb{E}\left|x_{k}(t)-x_{l}(t)\right|^{2}=0
$$

holds for any $k, l \in\{1,2, \ldots, N\}$.

In this paper, we aim to deal with the synchronization problem of the system (8) coupled by an array of $N$ identical delayed neutral-type neural networks with Markovian jumping parameters. The coupled networks involve both modedependent discrete time-delays and distributed time-delays with the mode-dependent upper bound. The coupled matrices are allowed to be mode-dependent as well. By constructing novel Lyapunov-Krasovskii functionals and using some analytical techniques, we shall derive easy-to-verify sufficient conditions to guarantee the coupled system to be asymptotically synchronized in mean square. The obtained criteria are given in terms of matrix inequalities that can be solved efficiently by employing the semi-definite programme method.

\section{MAin RESUltS AND PROOFS}

Before stating our main results, we introduce the following lemmas.

Lemma 1 ( [20]): Let $\mathcal{U}=\left(\alpha_{i j}\right)_{N \times N}, P \in \mathbb{R}^{n \times n}, x=$ $\left(x_{1}^{T}, x_{2}^{T}, \ldots, x_{N}^{T}\right)^{T}$, and $y=\left(y_{1}^{T}, y_{2}^{T}, \ldots, y_{N}^{T}\right)^{T}$ with $x_{i}, y_{i} \in$ $\mathbb{R}^{n}$. If $\mathcal{U}=\mathcal{U}^{T}$ and each row sum of $\mathcal{U}$ is zero, then

$$
x^{T}(\mathcal{U} \otimes P) y=-\sum_{1 \leq i<j \leq N} \alpha_{i j}\left(x_{i}-x_{j}\right) P\left(y_{i}-y_{j}\right) .
$$

Lemma 2 ( [20]): Suppose that $\mathcal{B}=\operatorname{diag}\left\{\beta_{1}, \beta_{2}, \ldots, \beta_{n}\right\}$ is a positive semi-definite diagonal matrix. Let $y=$ $\left(y_{1}, y_{2}, \ldots, y_{n}\right)^{T} \in \mathbb{R}^{n}$, and $\mathcal{H}(y)=\left(\hbar_{1}\left(y_{1}\right), \hbar_{2}\left(y_{2}\right), \ldots\right.$, $\left.\hbar_{n}\left(y_{n}\right)\right)^{T}$ be a continuous nonlinear function satisfying

$$
l_{i}^{-} \leq \frac{\hbar_{i}(s)}{s} \leq l_{i}^{+}, s \neq 0, s \in \mathbb{R}, \quad i=1,2, \ldots, n
$$

with $l_{i}^{-}$and $l_{i}^{+}$being constant scalars. Then

$$
y^{T} \mathcal{B} L_{1} y-2 y^{T} \mathcal{B} L_{2} \mathcal{H}(y)+\mathcal{H}^{T}(y) \mathcal{B H}(y) \leq 0
$$

where $L_{1}=\operatorname{diag}\left\{l_{1}^{+} l_{1}^{-}, l_{2}^{+} l_{2}^{-}, \ldots, l_{n}^{+} l_{n}^{-}\right\}$and $L_{2}=$ $\operatorname{diag}\left\{\frac{l_{1}^{+}+l_{1}^{-}}{2}, \frac{l_{2}^{+}+l_{2}^{-}}{2}, \ldots, \frac{l_{n}^{+}+l_{n}^{-}}{2}\right\}$. 


$$
\Phi_{k l}(i)=\left(\begin{array}{cccccccc}
\Xi_{11}(i) & \Xi_{12}(i) & \Theta_{i} \Sigma_{2} & \Xi_{14}(i) & \Omega_{i} \Upsilon_{2} & \Xi_{16}(i) & \Xi_{17} & -\sqrt{\kappa_{1}} A(i) Q \\
* & -\Lambda_{i} & 0 & 0 & 0 & 0 & 0 & \sqrt{\kappa_{1}} B^{T}(i) Q \\
* & * & \Xi_{33}(i) & 0 & 0 & 0 & 0 & 0 \\
* & * & * & -R & 0 & 0 & 0 & \sqrt{\kappa_{1}} C^{T}(i) Q \\
* & * & * & * & \Xi_{55}(i) & 0 & 0 & 0 \\
* & * & * & * & * & -\frac{1}{\alpha_{i}} S & 0 & \sqrt{\kappa_{1}} D^{T}(i) Q \\
* & * & * & * & * & * & -Q & \sqrt{\kappa_{1}} E^{T}(i) Q \\
* & * & * & * & * & * & * & -Q
\end{array}\right)<0,
$$

Lemma 3 ( [22]): Let $M$ be a positive semi-definite matrix, $\alpha(\cdot):(-\infty, a] \rightarrow[0,+\infty)$ be a scalar function and $\mathcal{F}(\cdot):(-\infty, a] \rightarrow \mathbb{R}^{n}$ be a vector function. If the integrations concerned are well defined, the following inequality holds:

$$
\begin{aligned}
& \left(\int_{-\infty}^{a} \alpha(s) \mathcal{F}(s) d s\right)^{T} M\left(\int_{-\infty}^{a} \alpha(s) \mathcal{F}(s) d s\right) \\
\leq & \int_{-\infty}^{a} \alpha(s) d s\left(\int_{-\infty}^{a} \alpha(s) \mathcal{F}^{T}(s) M \mathcal{F}(s) d s\right) .
\end{aligned}
$$

Lemma 4 (Schur Complement [6]): Given constant matrices $\Omega_{1}, \Omega_{2}, \Omega_{3}$ where $\Omega_{1}=\Omega_{1}^{T}$ and $\Omega_{2}>0$, then

$$
\Omega_{1}+\Omega_{3}^{T} \Omega_{2}^{-1} \Omega_{3}<0
$$

if and only if

$$
\left[\begin{array}{cc}
\Omega_{1} & \Omega_{3}^{T} \\
\Omega_{3} & -\Omega_{2}
\end{array}\right]<0
$$

Lemma 5 (Barbalat's Lemma [32]): Let $f$ be a nonnegative function defined on $[0,+\infty)$. If $f$ is Lebesgue integrable on $[0,+\infty)$ and is uniformly continuous on $[0,+\infty)$, then $\lim _{t \rightarrow+\infty} f(t)=0$.

For presentation convenience, in the following, we denote

$$
\begin{aligned}
L_{1} & =\operatorname{diag}\left\{l_{1}^{+} l_{1}^{-}, \ldots, l_{n}^{+} l_{n}^{-}\right\}, \\
L_{2} & =\operatorname{diag}\left\{\frac{l_{1}^{+}+l_{1}^{-}}{2}, \ldots, \frac{l_{n}^{+}+l_{n}^{-}}{2}\right\}, \\
\Sigma_{1} & =\operatorname{diag}\left\{\sigma_{1}^{+} \sigma_{1}^{-}, \ldots, \sigma_{n}^{+} \sigma_{n}^{-}\right\}, \\
\Sigma_{2} & =\operatorname{diag}\left\{\frac{\sigma_{1}^{+}+\sigma_{1}^{-}}{2}, \ldots, \frac{\sigma_{n}^{+}+\sigma_{n}^{-}}{2}\right\}, \\
\Upsilon_{1} & =\operatorname{diag}\left\{v_{1}^{+} v_{1}^{-}, \ldots, v_{n}^{+} v_{n}^{-}\right\}, \\
\Upsilon_{2} & =\operatorname{diag}\left\{\frac{v_{1}^{+}+v_{1}^{-}}{2}, \ldots, \frac{v_{n}^{+}+v_{n}^{-}}{2}\right\}, \\
\bar{\tau}_{1} & =\max _{1 \leq j \leq n_{0}}\left\{\tau_{1, j}\right\}, \bar{\tau}_{2}=\max _{1 \leq j \leq n_{0}}\left\{\tau_{2, j}\right\}, \bar{\tau}_{3}=\max _{1 \leq j \leq n_{0}}\left\{\tau_{3, j}\right\}, \\
\underline{\tau}_{1} & =\min _{1 \leq j \leq n_{0}}\left\{\tau_{1, j}\right\}, \underline{\tau}_{2}=\min _{1 \leq j \leq n_{0}}\left\{\tau_{2, j}\right\}, \underline{\tau}_{3}=\min _{1 \leq j \leq n_{0}}\left\{\tau_{3, j}\right\}, \\
\bar{\pi} & =\max _{1 \leq i \leq n_{0}}\left\{\left|\pi_{i i}\right|\right\} .
\end{aligned}
$$

The main results of this paper are given in the following theorem.

Theorem 1: Under Assumptions 1-3, the system (8) is globally asymptotically synchronized in mean square if there exist six positive definite matrices $P_{1}, P_{2}, P_{3}, Q, R$ and $S$, and three sets of positive definite diagonal matrices $\Lambda_{i}, \Theta_{i}$ and $\Omega_{i}\left(1 \leq i \leq n_{0}\right)$ such that the following LMIs (11) shown at the top of the page hold for $1 \leq k<l \leq N, 1 \leq i \leq n_{0}$, where

$$
\begin{aligned}
& \bar{\varphi}=\bar{\pi} \sup _{\underline{\tau}_{3} \leq s \leq \bar{\tau}_{3}} \varphi(s), \alpha_{i}=\int_{\tau_{3, i}}^{+\infty} \varphi(s) d s, \\
& \hat{\alpha}_{i}=\alpha_{i}+\frac{1}{2} \bar{\varphi}\left(\bar{\tau}_{3}^{2}-\underline{\tau}_{3}^{2}\right), \kappa_{1}=\bar{\pi}\left(\bar{\tau}_{1}-\underline{\tau}_{1}\right)+1, \\
& \Xi_{11}(i)=-P_{i} A(i)-A(i) P_{i}+\bar{P}_{i}-N w_{k l}(i)\left(P_{i} \Gamma(i)\right. \\
& \left.+\Gamma(i) P_{i}\right)-\left(\Lambda_{i} L_{1}+\Theta_{i} \Sigma_{1}+\Omega_{i} \Upsilon_{1}\right) \\
& +\kappa_{1} N w_{k l}(i)(\Gamma(i) Q A(i)+A(i) Q \Gamma(i)) \\
& -\kappa_{1} N w_{k l}^{(2)}(i) \Gamma(i) Q \Gamma(i) \text {, } \\
& \Xi_{12}(i)=P_{i} B(i)+\Lambda_{i} L_{2}-\kappa_{1} N w_{k l}(i) \Gamma(i) Q B(i), \\
& \Xi_{33}(i)=\left[\bar{\pi}\left(\bar{\tau}_{2}-\underline{\tau}_{2}\right)+1\right] R-\Theta_{i}, \\
& \Xi_{14}(i)=P_{i} C(i)-\kappa_{1} N w_{k l}(i) \Gamma(i) Q C(i), \\
& \Xi_{55}(i)=\hat{\alpha}_{i} S-\Omega_{i} \text {, } \\
& \Xi_{16}(i)=P_{i} D(i)-\kappa_{1} N w_{k l}(i) \Gamma(i) Q D(i), \\
& \Xi_{17}(i)=P_{i} E(i)-\kappa_{1} N w_{k l}(i) \Gamma(i) Q E(i),
\end{aligned}
$$

and $w_{k l}^{(2)}(i)$ is the $(k, l)$-th entry of matrix $[W(i)]^{2}$.

Proof: Define $x_{t}(\cdot)$ by $x_{t}(s)=x(t+s)(-\infty<s \leq 0)$ and denote

$$
U=\left[\begin{array}{cccc}
N-1 & -1 & \cdots & -1 \\
-1 & N-1 & \cdots & -1 \\
\cdots & \cdots & \cdots & \cdots \\
-1 & -1 & \cdots & N-1
\end{array}\right]_{N \times N}
$$

In order to tackle the synchronization problem of (8), we introduce the following Lyapunov-Krasovskii functional candidate:

$$
V\left(x_{t}, t, r(t)\right):=\sum_{k=1}^{6} V_{k}\left(x_{t}, t, r(t)\right)
$$

where

$$
\begin{aligned}
& V_{1}\left(x_{t}, t, r(t)\right)=x^{T}(t)\left(U \otimes P_{r(t)}\right) x(t), \\
& V_{2}\left(x_{t}, t, r(t)\right)=\int_{t-\tau_{1, r(t)}}^{t} \dot{x}^{T}(s)(U \otimes Q) \dot{x}(s) d s, \\
& V_{3}\left(x_{t}, t, r(t)\right)=\int_{t-\tau_{2, r(t)}}^{t} \mathbf{g}^{T}(x(s))(U \otimes R) \mathbf{g}(x(s)) d s, \\
& V_{4}\left(x_{t}, t, r(t)\right)=\bar{\pi} \int_{\underline{\tau}_{1}}^{\bar{\tau}_{1}} \int_{t-s}^{t} \dot{x}^{T}(\theta)(U \otimes Q) \dot{x}(\theta) d \theta d s \\
&+\bar{\pi} \int_{\underline{\tau}_{2}}^{\bar{\tau}_{2}} \int_{t-s}^{t} \mathbf{g}^{T}(x(\theta))(U \otimes R) \mathbf{g}(x(\theta)) d \theta d s,
\end{aligned}
$$




$$
\begin{aligned}
V_{5}\left(x_{t}, t, r(t)\right)= & \int_{\tau_{3, r(t)}}^{+\infty} \varphi(s) \int_{t-s}^{t} \mathbf{h}^{T}(x(\eta)) \\
& \times(U \otimes S) \mathbf{h}(x(\eta)) d \eta d s, \\
V_{6}\left(x_{t}, t, r(t)\right)= & \bar{\varphi} \int_{\underline{\tau}_{3}}^{\bar{\tau}_{3}} \int_{0}^{u} \int_{t-s}^{t} \mathbf{h}^{T}(x(\eta)) \\
& \times(U \otimes S) \mathbf{h}(x(\eta)) d \eta d s d u
\end{aligned}
$$

with $\bar{\varphi}$ defined in (12).

Let $\mathscr{L}$ be the weak infinitesimal generator of the random process $\left\{\left(x_{t}, r(t)\right), t \geq 0\right\}$ along the network (8) defined by

$$
\begin{gathered}
\mathscr{L} V\left(x_{t}, t, i\right)=\lim _{\Delta \rightarrow 0^{+}} \frac{1}{\Delta}\left[\mathbb { E } \left[V\left(x_{t+\Delta}, t+\Delta, r(t+\Delta)\right) \mid x_{t},\right.\right. \\
\left.r(t)=i]-V\left(x_{t}, t, i\right)\right] .
\end{gathered}
$$

Then, we have

$$
\mathscr{L} V\left(x_{t}, t, i\right)=\sum_{k=1}^{6} \mathscr{L} V_{k}\left(x_{t}, t, i\right)
$$

where $\mathscr{L} V_{k}\left(x_{t}, t, i\right) \quad(k=1,2, \cdots, 6)$ are calculated as follows.

First of all, it follows that

$$
\begin{aligned}
& \mathscr{L} V_{1}\left(x_{t}, t, i\right) \\
&=2 x^{T}(t)\left(U \otimes P_{i}\right)\left[\left(I_{N} \otimes E(i)\right) \dot{x}\left(t-\tau_{1, r(t)}\right)\right. \\
&-\left(I_{N} \otimes A(i)\right) x(t)+\left(I_{N} \otimes B(i)\right) \mathbf{f}(x(t)) \\
&+\left(I_{N} \otimes C(i)\right) \mathbf{g}\left(x\left(t-\tau_{2, i}\right)\right)+\left(I_{N} \otimes D(i)\right) \\
& \times \int_{-\infty}^{t-\tau_{3, i}} \varphi(t-s) \mathbf{h}(x(s)) d s+\mathbf{u}(t) \\
&+W(i) \otimes \Gamma(i) x(t)]+\sum_{j=1}^{n_{0}} \pi_{i j} x^{T}(t)\left(U \otimes P_{j}\right) x(t) \\
&=2 x^{T}(t)\left[\left(U \otimes\left(P_{i} E(i)\right)\right) \dot{x}\left(t-\tau_{1, r}(t)\right)\right. \\
&-\left(U \otimes\left(P_{i} A(i)\right)\right) x(t)+\left(U \otimes\left(P_{i} B(i)\right)\right) \mathbf{f}(x(t)) \\
&+\left(U \otimes\left(P_{i} C(i)\right)\right) \mathbf{g}\left(x\left(t-\tau_{2, i}\right)\right)+\left(U \otimes\left(P_{i} D(i)\right)\right) \\
&\left.\quad \int_{-\infty}^{t-\tau_{3, i}} \varphi(t-s) \mathbf{h}(x(s)) d s+N W(i) \otimes\left(P_{i} \Gamma(i)\right) x(t)\right] \\
&+x^{T}(t)\left(U \otimes \bar{P}_{i}\right) x(t),
\end{aligned}
$$

where we have used the facts that $U W(i)=N W(i)$ and $U \otimes \mathbf{u}(t)=0$, which are not difficult to verify.

Next, it can be obtained that

$$
\begin{aligned}
& \mathscr{L} V_{2}\left(x_{t}, t, i\right) \\
=\quad & \dot{x}^{T}(t)(U \otimes Q) \dot{x}(t)-\dot{x}^{T}\left(t-\tau_{1, i}\right)(U \otimes Q) \dot{x}\left(t-\tau_{1, i}\right) \\
& +\sum_{j=1}^{n_{0}} \pi_{i j} \int_{t-\tau_{1, j}}^{t} \dot{x}^{T}(s)(U \otimes Q) \dot{x}(s) d s \\
=\quad & \dot{x}^{T}(t)(U \otimes Q) \dot{x}(t)-\dot{x}^{T}\left(t-\tau_{1, i}\right)(U \otimes Q) \dot{x}\left(t-\tau_{1, i}\right) \\
& +\sum_{j \neq i}^{n_{0}} \pi_{i j}\left[\int_{t-\tau_{1, i}}^{t}+\int_{t-\tau_{1, j}}^{t-\tau_{1, i}}\right] \dot{x}^{T}(s)(U \otimes Q) \dot{x}(s) d s \\
& +\pi_{i i} \int_{t-\tau_{1, i}}^{t} \dot{x}^{T}(s)(U \otimes Q) \dot{x}(s) d s \\
\leq \quad & \dot{x}^{T}(t)(U \otimes Q) \dot{x}(t)-\dot{x}^{T}\left(t-\tau_{1, i}\right)(U \otimes Q) \dot{x}\left(t-\tau_{1, i}\right)
\end{aligned}
$$

$$
+\bar{\pi} \int_{t-\bar{\tau}_{1}}^{t-\underline{\tau}_{1}} \dot{x}^{T}(s)(U \otimes Q) \dot{x}(s) d s .
$$

Similar to (24), it follows that

$$
\begin{aligned}
\mathscr{L} V_{3}\left(x_{t}, t, i\right) \leq \mathbf{g}^{T}( & x(t))(U \otimes R) \mathbf{g}(x(t)) \\
& -\mathbf{g}^{T}\left(x\left(t-\tau_{2, i}\right)\right)(U \otimes R) \mathbf{g}\left(x\left(t-\tau_{2, i}\right)\right) \\
& +\bar{\pi} \int_{t-\bar{\tau}_{2}}^{t-\overline{\mathbf{T}}^{2}}(x(s))(U \otimes R) \mathbf{g}(x(s)) d s .
\end{aligned}
$$

It is easy to see

$$
\begin{aligned}
\mathscr{L} V_{4}\left(x_{t}, t, i\right)=\bar{\pi} & \left(\bar{\tau}_{1}-\underline{\tau}_{1}\right) \dot{x}^{T}(t)(U \otimes Q) \dot{x}(t) \\
& -\bar{\pi} \int_{t-\bar{\tau}_{1}}^{t-\underline{\tau}_{1}} \dot{x}^{T}(s)(U \otimes Q) \dot{x}(s) d s \\
& +\bar{\pi}\left(\bar{\tau}_{2}-\underline{\tau}_{2}\right) \mathbf{g}^{T}(x(t))(U \otimes R) \mathbf{g}(x(t)) \\
& -\bar{\pi} \int_{t-\bar{\tau}_{2}}^{t-\overline{\mathbf{g}}_{2}}(x(s))(U \otimes R) \mathbf{g}(x(s)) d s .(26)
\end{aligned}
$$

Then, it follows

$$
\begin{aligned}
& \mathscr{L} V_{5}\left(x_{t}, t, i\right) \\
& =\int_{\tau_{3, i}}^{+\infty} \varphi(s) d s \mathbf{h}^{T}(x(t))(U \otimes S) \mathbf{h}(x(t)) \\
& -\int_{\tau_{3, i}}^{+\infty} \varphi(s) \mathbf{h}^{T}(x(t-s))(U \otimes S) \mathbf{h}(x(t-s)) d s \\
& +\sum_{j=1}^{n_{0}} \pi_{i j} \int_{\tau_{3, j}}^{+\infty} \varphi(s) \int_{t-s}^{t} \mathbf{h}^{T}(x(\eta))(U \otimes S) \mathbf{h}(x(\eta)) d \eta d s \\
& =\alpha_{i} \mathbf{h}^{T}(x(t))(U \otimes S) \mathbf{h}(x(t)) \\
& -\int_{-\infty}^{t-\tau_{3, i}} \varphi(t-s) \mathbf{h}^{T}(x(s))(U \otimes S) \mathbf{h}(x(s)) d s \\
& +\sum_{j \neq i} \pi_{i j} \int_{\tau_{3, j}}^{+\infty} \varphi(s) \int_{t-s}^{t} \mathbf{h}^{T}(x(\eta))(U \otimes S) \mathbf{h}(x(\eta)) d \eta d s \\
& +\pi_{i i} \int_{\tau_{3, i}}^{+\infty} \varphi(s) \int_{t-s}^{t} \mathbf{h}^{T}(x(\eta))(U \otimes S) \mathbf{h}(x(\eta)) d \eta d s \\
& =\alpha_{i} \mathbf{h}^{T}(x(t))(U \otimes S) \mathbf{h}(x(t)) \\
& -\int_{-\infty}^{t-\tau_{3, i}} \varphi(t-s) \mathbf{h}^{T}(x(s))(U \otimes S) \mathbf{h}(x(s)) d s \\
& +\sum_{j \neq i} \pi_{i j} \int_{\tau_{3, j}}^{\tau_{3, i}} \varphi(s) \int_{t-s}^{t} \mathbf{h}^{T}(x(\eta))(U \otimes S) \mathbf{h}(x(\eta)) d \eta d s \\
& \leq \alpha_{i} \mathbf{h}^{T}(x(t))(U \otimes S) \mathbf{h}(x(t)) \\
& -\int_{-\infty}^{t-\tau_{3, i}} \varphi(t-s) \mathbf{h}^{T}(x(s))(U \otimes S) \mathbf{h}(x(s)) d s \\
& +\bar{\pi} \max _{\tau_{3} \leq s \leq \tau_{3}} \varphi(s) \int_{\underline{\tau}_{3}}^{\bar{\tau}_{3}} \int_{t-s}^{t} \mathbf{h}^{T}(x(\eta))(U \otimes S \mathbf{h}(x(\eta)) d \eta d s \\
& =\alpha_{i} \mathbf{h}^{T}(x(t))(U \otimes S) \mathbf{h}(x(t)) \\
& -\int_{-\infty}^{t-\tau_{3, i}} \varphi(t-s) \mathbf{h}^{T}(x(s))(U \otimes S) \mathbf{h}(x(s)) d s \\
& +\bar{\varphi} \int_{\underline{\tau}_{3}}^{\bar{\tau}_{3}} \int_{t-s}^{t} \mathbf{h}^{T}(x(\eta))(U \otimes S) \mathbf{h}(x(\eta)) d \eta d s,
\end{aligned}
$$

where $\alpha_{i}$ is defined in (12). 
Finally, we have

$$
\begin{aligned}
& \mathscr{L} V_{6}\left(x_{t}, t, i\right) \\
= & \bar{\varphi} \int_{\underline{\tau}_{3}}^{\bar{\tau}_{3}} \int_{0}^{u} \mathbf{h}^{T}(x(t))(U \otimes S) \mathbf{h}(x(t)) d \eta d s d u \\
& -\bar{\varphi} \int_{\underline{\tau}_{3}}^{\bar{\tau}_{3}} \int_{0}^{u} \mathbf{h}^{T}(x(t-s))(U \otimes S) \mathbf{h}(x(t-s)) d s d u \\
= & \frac{1}{2} \bar{\varphi}\left(\bar{\tau}_{3}^{2}-\underline{\tau}_{3}^{2}\right) \mathbf{h}^{T}(x(t))(U \otimes S) \mathbf{h}(x(t)) \\
& -\bar{\varphi} \int_{\underline{\tau}_{3}}^{\bar{\tau}_{3}} \int_{t-s}^{t} \mathbf{h}^{T}(x(\eta))(U \otimes S) \mathbf{h}(x(\eta)) d \eta d s .
\end{aligned}
$$

Substituting (23)-(28) into (22) yields that

$$
\begin{aligned}
& \mathscr{L} V\left(x_{t}, t, i\right) \\
\leq 2 x^{T}(t)\left[\left(U \otimes\left(P_{i} E(i)\right)\right) \dot{x}\left(t-\tau_{1, r(t)}\right)\right. & \\
- & \left(U \otimes\left(P_{i} A(i)\right)\right) x(t)+\left(U \otimes\left(P_{i} B(i)\right)\right) \mathbf{f}(x(t)) \\
+ & \left(U \otimes\left(P_{i} C(i)\right)\right) \mathbf{g}\left(x\left(t-\tau_{2, i}\right)\right)+\left(U \otimes\left(P_{i} D(i)\right)\right) \\
& \left.\times \int_{-\infty}^{t-\tau_{3, i}} \varphi(t-s) \mathbf{h}(x(s)) d s+N W(i) \otimes\left(P_{i} \Gamma(i)\right) x(t)\right] \\
+ & x^{T}(t)\left(U \otimes \bar{P}_{i}\right) x(t)+\left[\bar{\pi}_{(}\left(\bar{\tau}_{1}-\underline{\tau}_{1}\right)+1\right] \dot{x}^{T}(t) \\
\times & (U \otimes Q) \dot{x}(t)-\dot{x}^{T}\left(t-\tau_{1, i}\right)(U \otimes Q) \dot{x}\left(t-\tau_{1, i}\right) \\
+ & {\left[\bar{\pi}\left(\bar{\tau}_{2}-\underline{\tau}_{2}\right)+1\right] \mathbf{g}^{T}(x(t))(U \otimes R) \mathbf{g}(x(t)) } \\
- & \mathbf{g}^{T}\left(x\left(t-\tau_{2, i}\right)\right)(U \otimes R) \mathbf{g}\left(x\left(t-\tau_{2, i}\right)\right) \\
+ & \hat{\alpha}_{i} \mathbf{h}^{T}(x(t))(U \otimes S) \mathbf{h}(x(t)) \\
- & \int_{-\infty}^{t-\tau_{3, i}} \varphi(t-s) \mathbf{h}^{T}(x(s))(U \otimes S) \mathbf{h}(x(s)) d s,
\end{aligned}
$$

where $\hat{\alpha}_{i}$ is defined in (13).

For the sake of the presentation simplicity, we also denote

$$
\mathbf{x}_{k l}(t)=x_{k}(t)-x_{l}(t), \quad \overline{\mathbf{f}}_{k l}(t)=f\left(x_{k}(t)\right)-f\left(x_{l}(t)\right),
$$

$\overline{\mathbf{g}}_{k l}(t)=g\left(x_{k}(t)\right)-g\left(x_{l}(t)\right), \quad \overline{\mathbf{h}}_{k l}(t)=h\left(x_{k}(t)\right)-h\left(x_{l}(t)\right)$.

By applying Lemma 1 to (29), we have

$$
\begin{aligned}
& \mathscr{L} V\left(x_{t}, t, i\right) \\
= & \sum_{1 \leq k<l \leq N} 2 \mathbf{x}_{k l}^{T}(t)\left[P_{i} E(i) \dot{\mathbf{x}}_{k l}\left(t-\tau_{1, i}\right)-P_{i} A(i) \mathbf{x}_{k l}(t)\right. \\
& +P_{i} B(i) \overline{\mathbf{f}}_{k l}(t)+P_{i} C(i) \overline{\mathbf{g}}_{k l}\left(t-\tau_{2, i}\right)+P_{i} D(i) \\
& \left.\times \int_{-\infty}^{t-\tau_{3, i}} \varphi(t-s) \overline{\mathbf{h}}_{k l}(s) d s-N w_{k l}(i) P_{i} \Gamma(i) \mathbf{x}_{k l}(t)\right] \\
& +\sum_{1 \leq k<l \leq N}\left[\mathbf{x}_{k l}^{T}(t) \bar{P}_{i} \mathbf{x}_{k l}(t)-\dot{\mathbf{x}}_{k l}^{T}\left(t-\tau_{1, i}\right) Q \dot{\mathbf{x}}_{k l}\left(t-\tau_{1, i}\right)\right. \\
& \left.+\left[\bar{\pi}_{\bar{\tau}}-\underline{\tau}_{2}\right)+1\right] \overline{\mathbf{g}}_{k l}^{T}(t) R \overline{\mathbf{g}}_{k l}(t) \\
& -\overline{\mathbf{g}}_{k l}^{T}\left(t-\tau_{2, i}\right) R \overline{\mathbf{g}}_{k l}\left(t-\tau_{2, i}\right)+\hat{\alpha}_{i} \overline{\mathbf{h}}_{k l}^{T}(t) S \overline{\mathbf{h}}_{k l}(t) \\
& \left.-\int_{-\infty}^{t-\tau_{3, i}} \varphi(t-s) \overline{\mathbf{h}}_{k l}^{T}(s) S \overline{\mathbf{h}}_{k l}(s) d s\right] \\
& +\left[\bar{\pi}\left(\bar{\tau}_{1}-\underline{\tau}_{1}\right)+1\right] \dot{x}^{T}(t)(U \otimes Q) \dot{x}(t) .
\end{aligned}
$$

From Assumption 2 and Lemma 2, we can deduce that

$$
\begin{aligned}
& \quad \mathbf{x}_{k l}^{T}(t) \Lambda_{i} L_{1} \mathbf{x}_{k l}(t)-2 \mathbf{x}_{k l}^{T}(t) \Lambda_{i} L_{2} \overline{\mathbf{f}}_{k l}(t) \\
& +\overline{\mathbf{f}}_{k l}^{T}(t) \Lambda_{i} \overline{\mathbf{f}}_{k l}(t) \leq 0, \\
& \quad \mathbf{x}_{k l}^{T}(t) \Theta_{i} \Sigma_{1} \mathbf{x}_{k l}(t)-2 \mathbf{x}_{k l}^{T}(t) \Theta_{i} \Sigma_{2} \overline{\mathbf{g}}_{k l}(t) \\
& +\overline{\mathbf{g}}_{k l}^{T}(t) \Theta_{i} \overline{\mathbf{g}}_{k l}(t) \leq 0, \\
& \quad \mathbf{x}_{k l}^{T}(t) \Omega_{i} \Upsilon_{1} \mathbf{x}_{k l}(t)-2 \mathbf{x}_{k l}^{T}(t) \Omega_{i} \Upsilon_{2} \overline{\mathbf{h}}_{k l}(t) \\
& +\overline{\mathbf{h}}_{k l}^{T}(t) \Omega_{i} \overline{\mathbf{h}}_{k l}(t) \leq 0 .
\end{aligned}
$$

Also, in terms of Lemma 3 , it is easy to see that

$$
\begin{aligned}
& \int_{-\infty}^{t-\tau_{3, i}} \varphi(t-s) \overline{\mathbf{h}}_{k l}^{T}(s) S \overline{\mathbf{h}}_{k l}(s) d s \\
& \geq \frac{1}{\int_{-\infty}^{t-\tau_{3, i}} \varphi(t-s) d s} \int_{-\infty}^{t-\tau_{3, i}} \varphi(t-s) \overline{\mathbf{h}}_{k l}^{T}(s) d s S \\
& \times \int_{-\infty}^{t-\tau_{3, i}} \varphi(t-s) \overline{\mathbf{h}}_{k l}(s) d s \\
&=\frac{1}{\alpha_{i}} \int_{-\infty}^{t-\tau_{3, i}} \varphi(t-s) \overline{\mathbf{h}}_{k l}^{T}(s) d s S \int_{-\infty}^{t-\tau_{3, i}} \varphi(t-s) \overline{\mathbf{h}}_{k l}(s) d s
\end{aligned}
$$

From (30)-(34), it follows that

$$
\begin{aligned}
& \mathscr{L} V\left(x_{t}, t, i\right) \\
\leq & \sum_{1 \leq k<l \leq N} 2 \mathbf{x}_{k l}^{T}(t)\left[P_{i} E(i) \dot{\mathbf{x}}_{k l}\left(t-\tau_{1, i}\right)-P_{i} A(i) \mathbf{x}_{k l}(t)\right. \\
+ & P_{i} B(i) \overline{\mathbf{f}}_{k l}(t)+P_{i} C(i) \overline{\mathbf{g}}_{k l}\left(t-\tau_{2, i}\right)+P_{i} D(i) \\
\times & \left.\int_{-\infty}^{t-\tau_{3, i}} \varphi(t-s) \overline{\mathbf{h}}_{k l}(s) d s-N w_{k l}(i) P_{i} \Gamma(i) \mathbf{x}_{k l}(t)\right] \\
+ & \sum_{1 \leq k<l \leq N}\left[\mathbf{x}_{k l}^{T}(t) \bar{P}_{i} \mathbf{x}_{k l}(t)-\dot{\mathbf{x}}_{k l}^{T}\left(t-\tau_{1, i}\right) Q \dot{\mathbf{x}}_{k l}\left(t-\tau_{1, i}\right)\right. \\
+ & {\left[\bar{\pi}\left(\bar{\tau}_{2}-\underline{\tau}_{2}\right)+1\right] \overline{\mathbf{g}}_{k l}^{T}(t) R \overline{\mathbf{g}}_{k l}(t)-\overline{\mathbf{g}}_{k l}^{T}\left(t-\tau_{2, i}\right) R } \\
\times & \overline{\mathbf{g}}_{k l}\left(t-\tau_{2, i}\right)+\hat{\alpha}_{i} \overline{\mathbf{h}}_{k l}^{T}(t) S \overline{\mathbf{h}}_{k l}(t) \\
- & \left.\frac{1}{\alpha_{i}} \int_{-\infty}^{t-\tau_{3, i}} \varphi(t-s) \overline{\mathbf{h}}_{k l}^{T}(s) d s S \int_{-\infty}^{t-\tau_{3, i}} \varphi(t-s) \overline{\mathbf{h}}_{k l}(s) d s\right] \\
- & \sum_{1 \leq k<l \leq N}\left[\mathbf{x}_{k l}^{T}(t)\left(\Lambda_{i} L_{1}+\Theta_{i} \Sigma_{1}+\Omega_{i} \Upsilon_{1}\right) \mathbf{x}_{k l}(t)\right. \\
- & 2 \mathbf{x}_{k l}^{T}(t) \Lambda_{i} L_{2} \overline{\mathbf{f}}_{k l}(t)+\overline{\mathbf{f}}_{k l}^{T}(t) \Lambda_{i} \overline{\mathbf{f}}_{k l}(t)-2 \mathbf{x}_{k l}^{T}(t) \Theta_{i} \Sigma_{2} \overline{\mathbf{g}}_{k l}(t) \\
+ & \left.\overline{\mathbf{g}}_{k l}^{T}(t) \Theta_{i} \overline{\mathbf{g}}_{k l}(t)-2 \mathbf{x}_{k l}^{T}(t) \Omega_{i} \Upsilon_{2} \overline{\mathbf{h}}_{k l}(t)+\overline{\mathbf{h}}_{k l}^{T}(t) \Omega_{i} \overline{\mathbf{h}}_{k l}(t)\right] \\
+ & {\left.\left[\bar{\pi}\left(\bar{\tau}_{1}-\underline{\tau}_{1}\right)+1\right] \dot{x}^{T}(t)(U) \otimes Q\right) \dot{x}(t), }
\end{aligned}
$$

where $\kappa_{1}$ is defined in (13).

For the last term in the above inequality, we have

$$
\begin{aligned}
& \dot{x}^{T}(t)(U \otimes Q) \dot{x}(t) \\
=\quad & {\left[\left(I_{N} \otimes E(i)\right) \dot{x}\left(t-\tau_{1, i}\right)-\left(I_{N} \otimes A(i)\right) x(t)\right.} \\
& +\left(I_{N} \otimes B(i)\right) \mathbf{f}(x(t))+\left(I_{N} \otimes C(i)\right) \mathbf{g}\left(x\left(t-\tau_{2, i}\right)\right) \\
& +\left(I_{N} \otimes D(i)\right) \int_{-\infty}^{t-\tau_{3, i}} \varphi(t-s) \mathbf{h}(x(s)) d s+\mathbf{u}(t) \\
& +W(i) \otimes \Gamma(i) x(t)]^{T}(U \otimes Q)\left[\left(I_{N} \otimes E(i)\right)\right. \\
& \times \dot{x}\left(t-\tau_{1, i}\right)-\left(I_{N} \otimes A(i)\right) x(t)
\end{aligned}
$$




$$
\begin{aligned}
& +\left(I_{N} \otimes B(i)\right) \mathbf{f}(x(t))+\left(I_{N} \otimes C(i)\right) \mathbf{g}\left(x\left(t-\tau_{2, i}\right)\right) \\
& +\left(I_{N} \otimes D(i)\right) \int_{-\infty}^{t-\tau_{3, i}} \varphi(t-s) \mathbf{h}(x(s)) d s+\mathbf{u}(t) \\
& +W(i) \otimes \Gamma(i) x(t)] \\
= & {\left[\left(I_{N} \otimes E(i)\right) \dot{x}\left(t-\tau_{1, i}\right)-\left(I_{N} \otimes A(i)\right) x(t)\right.} \\
& +\left(I_{N} \otimes B(i)\right) \mathbf{f}(x(t))+\left(I_{N} \otimes C(i)\right) \mathbf{g}\left(x\left(t-\tau_{2, i}\right)\right) \\
& \left.+\left(I_{N} \otimes D(i)\right) \int_{-\infty}^{t-\tau_{3, i}} \varphi(t-s) \mathbf{h}(x(s)) d s\right]^{T} \\
& \times(U \otimes Q)\left[\left(I_{N} \otimes E(i)\right) \dot{x}\left(t-\tau_{1, i}\right)\right. \\
& -\left(I_{N} \otimes A(i)\right) x(t)+\left(I_{N} \otimes B(i)\right) \mathbf{f}(x(t)) \\
& +\left(I_{N} \otimes C(i)\right) \mathbf{g}\left(x\left(t-\tau_{2, i}\right)\right)+\left(I_{N} \otimes D(i)\right) \\
& \left.\times \int_{-\infty}^{t-\tau_{3, i}} \varphi(t-s) \mathbf{h}(x(s)) d s\right]+2 x^{T}(t) \\
& \times(W(i) \otimes \Gamma(i))(U \otimes Q)\left[\left(I_{N} \otimes E(i)\right) \dot{x}\left(t-\tau_{1, i}\right)\right. \\
& -\left(I_{N} \otimes A(i)\right) x(t)+\left(I_{N} \otimes B(i)\right) \mathbf{f}(x(t)) \\
& +\left(I_{N} \otimes C(i)\right) \mathbf{g}\left(x\left(t-\tau_{2, i}\right)\right)+\left(I_{N} \otimes D(i)\right) \\
& \left.\times \int_{-\infty}^{t-\tau_{3, i}} \varphi(t-s) \mathbf{h}(x(s)) d s\right]+x^{T}(t)(W(i) \otimes \Gamma(i)) \\
& \times(U \otimes Q)(W(i) \otimes \Gamma(i)) x(t) .
\end{aligned}
$$

Noticing the relationships

$$
\begin{aligned}
& (W(i) \otimes \Gamma(i))(U \otimes Q)=N(W(i) \otimes(\Gamma(i) Q), \\
& (W(i) \otimes \Gamma(i))(U \otimes Q)(W(i) \otimes \Gamma(i)) \\
=\quad & N[W(i)]^{2} \otimes(\Gamma(i) Q \Gamma(i))
\end{aligned}
$$

we can infer from Lemma 1 that

$$
\begin{aligned}
& \dot{x}^{T}(t)(U \otimes Q) \dot{x}(t) \\
= & \sum_{1 \leq k<l \leq N}\left[E(i) \dot{\mathbf{x}}_{k l}\left(t-\tau_{1, i}\right)-A(i) \mathbf{x}_{k l}(t)\right. \\
& +B(i) \mathbf{\mathbf { f }}_{k l}(x(t))+C(i) \overline{\mathbf{g}}_{k l}\left(t-\tau_{2, i}\right) \\
& \left.+D(i) \int_{-\infty}^{t-\tau_{3, i}} \varphi(t-s) \overline{\mathbf{h}}_{k l}(s) d s\right]^{T} Q\left[E(i) \dot{\mathbf{x}}_{k l}\left(t-\tau_{1, i}\right)\right. \\
& -A(i) \mathbf{x}_{k l}(t)+B(i) \overline{\mathbf{f}}_{k l}(x(t))+C(i) \overline{\mathbf{g}}_{k l}\left(t-\tau_{2, i}\right) \\
& \left.+D(i) \int_{-\infty}^{t-\tau_{3, i}} \varphi(t-s) \overline{\mathbf{h}}_{k l}(s) d s\right]-2 N \sum_{1 \leq k<l \leq N} \mathbf{x}_{k l}^{T}(t) \\
& \times w_{k l}(i) \Gamma(i) Q\left[E(i) \dot{\mathbf{x}}_{k l}\left(t-\tau_{1, i}\right)-A(i) \mathbf{x}_{k l}(t)\right. \\
& +B(i) \overline{\mathbf{f}}_{k l}(x(t))+C(i) \overline{\mathbf{g}}_{k l}\left(t-\tau_{2, i}\right) \\
& \left.+D(i) \int_{-\infty}^{t-\tau_{3, i}} \varphi(t-s) \overline{\mathbf{h}}_{k l}(s) d s\right] \\
& -N \sum_{1 \leq k<l \leq N}^{\mathbf{x}_{k l}^{T}(t) w_{k l}^{(2)}(i) \Gamma(i) Q \Gamma(i) \mathbf{x}_{k l}(t) .}
\end{aligned}
$$

Substituting (37) into (35) leads to

$$
\begin{aligned}
& \mathscr{L} V\left(x_{t}, t, i=\sum_{1 \leq k<l \leq N} \chi_{k l}^{T}(t, i)\left[\Psi_{k l}(i)\right.\right. \\
&\left.+\quad \mathcal{A}^{T}(i) \kappa_{1} Q \mathcal{A}(i)\right] \chi_{k l}(t, i)
\end{aligned}
$$

where $\Psi_{k l}(i)$ is defined as

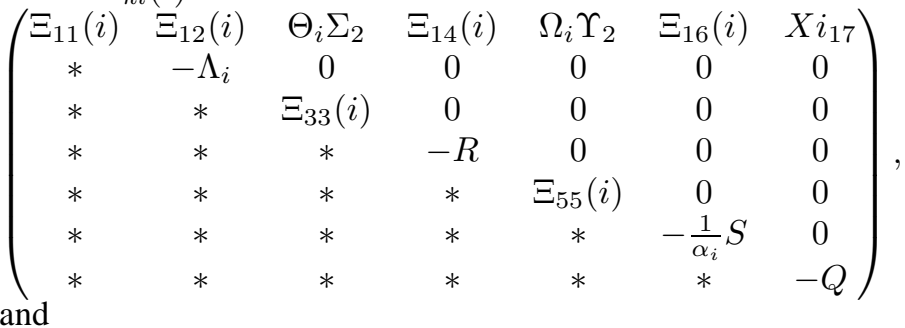

$$
\begin{aligned}
& \mathcal{A}(i)=\left[\begin{array}{lllllll}
-A(i) & B(i) & 0 & C(i) & 0 & D(i) & E(i)
\end{array}\right],
\end{aligned}
$$

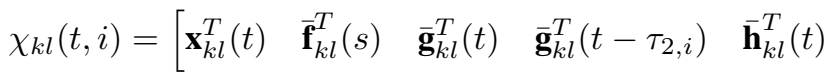

$$
\begin{aligned}
& \left.\int_{-\infty}^{t-\tau_{3, i}} \varphi(t-s) \overline{\mathbf{h}}_{k l}(s) d s \quad \dot{\mathbf{x}}_{k l}\left(t-\tau_{1, i}\right)\right]^{T} .
\end{aligned}
$$

In terms of Lemma 4, (11) is equivalent to

$$
\begin{aligned}
& \Psi_{k l}(i)+\mathcal{A}^{T}(i) \kappa_{1} Q \mathcal{A}(i)<0, \\
& \left(1 \leq k<l \leq N, 1 \leq i \leq n_{0}\right) .
\end{aligned}
$$

Let $\rho_{0}=\max \left\{\lambda_{\max }\left(\Psi_{k l}(i)+\mathcal{A}^{T}(i) \kappa_{1} Q \mathcal{A}(i)\right) \mid 1 \leq k<\right.$ $\left.l \leq N, 1 \leq i \leq n_{0}\right\}$. Obviously, $\rho_{0}<0$ and it then follows from (38) that

$$
\begin{aligned}
\mathscr{L} V\left(x_{t}, t, i\right) & \leq \rho_{0} \sum_{1 \leq k<l \leq N} \chi_{k l}^{T}(t, i) \chi_{k l}(t, i) \\
& \leq \rho_{0} \sum_{1 \leq k<l \leq N}\left|\mathbf{x}_{k l}(t)\right|^{2} .
\end{aligned}
$$

Therefore, we have

$$
\begin{aligned}
& \mathbb{E} V(x(t), t, r(t)) \\
= & \mathbb{E} V(x(0), 0, r(0))+\mathbb{E} \int_{0}^{t} L V(x(s), s, r(s)) d s \\
\leq & \mathbb{E} V(x(0), 0, r(0))+\rho_{0} \sum_{1 \leq k<l \leq N} \int_{0}^{t} \mathbb{E}\left|\mathbf{x}_{k l}(s)\right|^{2} d s .
\end{aligned}
$$

Since $\rho_{0}<0$ and $V(x(t), t, r(t))>0$, it follows readily from (41) that

$$
\sum_{1 \leq k<l \leq N} \int_{0}^{t} \mathbb{E}\left|\mathbf{x}_{k l}(s)\right|^{2} d s \leq \frac{1}{|\rho|} \mathbb{E} V(x(0), 0, r(0)),
$$

which implies that the integral $\sum_{1 \leq k<l \leq N} \int_{0}^{+\infty} \mathbb{E}\left|\mathbf{x}_{k l}(s)\right|^{2} d s<$ $+\infty$.

By Lemma 5, we have

$$
\lim _{t \rightarrow+\infty} \sum_{1 \leq k<l \leq N} \mathbb{E}\left|\mathbf{x}_{k l}(t)\right|^{2}=0,
$$

or $\lim _{t \rightarrow+\infty} \mathbb{E}\left|x_{k}(t)-x_{l}(t)\right|^{2}=0$ for $1 \leq k<l \leq N$. In other words, the system (8) is globally asymptotically synchronized in mean square. This completes the proof of the theorem.

The system (8) is rather general. In what follows, we consider two special cases. In Case 1, we show that our main results can be specialized to the synchronization problem for coupled system without involving the derivatives of the past history (i.e., $E(r(t))=0$ ), which reduces to a retarded 


$$
\hat{\Phi}_{k l}(i)=\left(\begin{array}{cccccc}
\hat{\Xi}_{11}(i) & P B(i)+\Lambda_{i} L_{2} & \Theta_{i} \Sigma_{2} & P C(i) & \Omega_{i} \Upsilon_{2} & P D(i) \\
* & -\Lambda_{i} & 0 & 0 & 0 & 0 \\
* & * & \Xi_{33}(i) & 0 & 0 & 0 \\
* & * & * & -R & 0 & 0 \\
* & * & * & * & \Xi_{55}(i) & 0 \\
* & * & * & * & * & -\frac{1}{\alpha_{i}} S
\end{array}\right)<0
$$

$$
\tilde{\Phi}(i)=\left(\begin{array}{cccccccc}
\tilde{\Xi}_{11}(i) & P_{i} B(i)+\Lambda_{i} L_{2} & \Theta_{i} \Sigma_{2} & P_{i} C(i) & \Omega_{i} \Upsilon_{2} & P_{i} D(i) & P_{i} E(i) & -\sqrt{\kappa_{1}} A(i) Q \\
* & -\Lambda_{i} & 0 & 0 & 0 & 0 & 0 & \sqrt{\kappa_{1}} B^{T}(i) Q \\
* & * & \Xi_{33}(i) & 0 & 0 & 0 & 0 & 0 \\
* & * & * & -R & 0 & 0 & 0 & \sqrt{\kappa_{1}} C^{T}(i) Q \\
* & * & * & * & \Xi_{55}(i) & 0 & 0 & 0 \\
* & * & * & * & * & -\frac{1}{\alpha_{i}} S & 0 & \sqrt{\kappa_{1}} D^{T}(i) Q \\
* & * & * & * & * & * & -Q & \sqrt{\kappa_{1}} E^{T}(i) Q \\
* & * & * & * & * & * & * & -Q
\end{array}\right)<0,
$$

functional differential equation. In Case 2, we consider the same array of neural networks with discrete time-delay only. to

Case 1. In the case of $E(r(t))=0$, the system (8) reduces

$$
\begin{aligned}
\dot{x}(t)=- & \left(I_{N} \otimes A(r(t))\right) x(t)+\left(I_{N} \otimes B(r(t))\right) \mathbf{f}(x(t)) \\
& +\left(I_{N} \otimes C(r(t))\right) \mathbf{g}\left(x\left(t-\tau_{2, i}\right)\right) \\
& +\left(I_{N} \otimes D(r(t))\right) \int_{-\infty}^{t-\tau_{3, r(t)}} \varphi(t-s) \mathbf{h}(x(s)) d s \\
& +\mathbf{u}(t)+W(r(t)) \otimes \Gamma(r(t)) x(t) .
\end{aligned}
$$

For the system (43), the following result can be derived based on Theorem 1.

Corollary 1: Under Assumptions 1-3, the system (43) is globally asymptotically synchronized in mean square if there exist five positive definite matrices $P_{1}, P_{2}, P_{3}, R$ and $S$, and three sets of positive definite diagonal matrices $\Lambda_{i}, \Theta_{i}$ and $\Omega_{i}\left(1 \leq i \leq n_{0}\right)$ such that the following LMIs (44) shown at the top of the page hold for $1 \leq k<l \leq N, 1 \leq i \leq n_{0}$, where each symbol has its previous meaning except $\hat{\Xi}_{11}(i)=$ $-P_{i} A(i)-A(i) P_{i}+\bar{P}_{i}-N w_{k l}(i)\left(P_{i} \Gamma(i)+\Gamma(i) P_{i}\right)-\left(\Lambda_{i} L_{1}+\right.$ $\left.\Theta_{i} \Sigma_{1}+\Omega_{i} \Upsilon_{1}\right)$.

Case 2. In this case, with $D(r(t))=0$, the system (8) is simplified as

$$
\begin{aligned}
\dot{x}(t)= & \left(I_{N} \otimes E(r(t))\right) \dot{x}\left(t-\tau_{1, r(t)}\right)-\left(I_{N} \otimes A(r(t))\right) x(t) \\
& +\left(I_{N} \otimes B(r(t))\right) \mathbf{f}(x(t))+\left(I_{N} \otimes C(r(t))\right) \\
& \times \mathbf{g}\left(x\left(t-\tau_{2, i}\right)\right)+\mathbf{u}(t) \\
& +W(r(t)) \otimes \Gamma(r(t)) x(t) .
\end{aligned}
$$

For the system (45), the following result is readily available.

Corollary 2: Under Assumptions 1-3, the system (45) is globally asymptotically synchronized in mean square if there exist five positive definite matrices $P_{1}, P_{2}, P_{3}, Q, R$ and $R$, and two sets of positive definite diagonal matrices $\Lambda_{i}$ and $\Theta_{i}\left(1 \leq i \leq n_{0}\right)$ such that the following LMIs

$$
\bar{\Phi}_{k l}(i)<0
$$

hold for $1 \leq k<l \leq N, 1 \leq i \leq n_{0}$, where $\bar{\Phi}_{k l}(i)$ is defined as

$$
\left(\begin{array}{cccccc}
\bar{\Xi}_{11}(i) & \Xi_{12}(i) & \Theta_{i} \Sigma_{2} & \Xi_{14}(i) & \Xi_{17} & -\sqrt{\kappa_{1}} A(i) Q \\
* & -\Lambda_{i} & 0 & 0 & 0 & \sqrt{\kappa_{1}} B^{T}(i) Q \\
* & * & \Xi_{33}(i) & 0 & 0 & 0 \\
* & * & * & -R & 0 & \sqrt{\kappa_{1}} C^{T}(i) Q \\
* & * & * & * & -Q & \sqrt{\kappa_{1}} E^{T}(i) Q \\
* & * & * & * & * & -Q
\end{array}\right),
$$

and each symbol has its previous meaning except $\bar{\Xi}_{11}(i)=$ $-P_{i} A(i)-A(i) P_{i}+\bar{P}_{i}-N w_{k l}(i)\left(P_{i} \Gamma(i)+\Gamma(i) P_{i}\right)-\left(\Lambda_{i} L_{1}+\right.$ $\left.\Theta_{i} \Sigma_{1}\right)$.

Remark 3: Notice that in the case of $W(i)=0$ or $\Gamma(i)=0$ for all $i$, the system (8) is uncoupled, and the dynamics of each single neutral-type network is independent of the other networks. Hence, by means of Theorem 1, a sufficient condition can be obtained to guarantee the global asymptotic stability in mean square for each single neutral-type neural network.

Corollary 3: Under Assumptions 1-3, the neutral-type neural network (1) is globally asymptotically stable in mean square if there exist six positive definite matrices $P_{1}, P_{2}$, $P_{3}, Q, R$ and $S$, and three sets of positive definite diagonal matrices $\Lambda_{i}, \Theta_{i}$ and $\Omega_{i}\left(1 \leq i \leq n_{0}\right)$ such that the following LMIs (46) shown at the top of the page hold for $1 \leq i \leq n_{0}$, where each symbol has its previous meaning except $\tilde{\Xi}_{11}(i)=$ $-P_{i} A(i)-A(i) P_{i}+\bar{P}_{i}-\left(\Lambda_{i} L_{1}+\Theta_{i} \Sigma_{1}+\Omega_{i} \Upsilon_{1}\right)$.

Remark 4: In this paper, the synchronization problem is dealt with for a new class of continuous-time neural networks of neutral-type with Markovian jumping parameters as well as mode-dependent mixed time-delays. Note that the mixed time-delays comprise both the discrete and distributed delays that are all dependent on the Markovian jumping mode. The novelty of the main results is fourfold: 1) due to the consideration of the mode-dependent neutral delays, some novel analysis techniques are developed to tackle the resulting mathematical difficulty; 2) a new Lyapunov functional is proposed to account for the Markovian jumps of the delay bounds; 3) a unified framework is established to handle the Markovian jumping parameters, neutral terms and mixed time-delays; and 4) the main results established in Theorem 1 contain all 
the information of the considered coupling neural networks including physical parameters, Markovian jumping rate, the discrete time-delay as well as bounds on the distributed timedelays. In the next section, a simulation example is provided to show the usefulness of the proposed stability conditions.

\section{A NUMERICAL EXAMPLE}

In this section, we present a simulation example so as to illustrate the usefulness of our main results. Our aim is to examine the global asymptotic synchronization of the system (8) in mean square.

Consider a system coupled by four identical second-order neutral-type neural networks with network parameters given as follows:

$$
\begin{aligned}
& \Pi=\left[\begin{array}{ccc}
-5 & 2 & 3 \\
4 & -5 & 1 \\
2 & 4 & -6
\end{array}\right], \quad A(1)=\left[\begin{array}{cc}
1 & 0 \\
0 & -0.3
\end{array}\right], \\
& B(1)=\left[\begin{array}{cc}
0.3 & 0.2 \\
0.2 & -0.1
\end{array}\right], \quad C(1)=\left[\begin{array}{cc}
0.3 & -0.1 \\
-0.4 & 0.1
\end{array}\right] \text {, } \\
& D(1)=\left[\begin{array}{cc}
0.3 & -0.2 \\
0.2 & 0.2
\end{array}\right], \quad E(1)=\left[\begin{array}{cc}
0.1 & 0.1 \\
0.1 & 0
\end{array}\right] \text {, } \\
& A(2)=\left[\begin{array}{cc}
1 & 0 \\
0 & -0.4
\end{array}\right], \quad B(2)=\left[\begin{array}{cc}
0.4 & 0.2 \\
0.2 & -0.2
\end{array}\right], \\
& C(2)=\left[\begin{array}{cc}
0.2 & -0.4 \\
0.2 & 0.2
\end{array}\right], D(2)=\left[\begin{array}{cc}
0.2 & -0.4 \\
0 & 0.2
\end{array}\right] \text {, } \\
& E(2)=\left[\begin{array}{ll}
0.1 & 0.1 \\
0.1 & 0.1
\end{array}\right], \quad A(3)=\left[\begin{array}{cc}
1 & 0 \\
0 & -0.5
\end{array}\right] \text {, } \\
& B(3)=\left[\begin{array}{cc}
0.2 & 0 \\
0.2 & -0.2
\end{array}\right], C(3)=\left[\begin{array}{cc}
0.2 & 0.3 \\
-0.3 & 0
\end{array}\right] \text {, } \\
& D(3)=\left[\begin{array}{cc}
0.1 & -0.2 \\
0.1 & 0.2
\end{array}\right], E(3)=\left[\begin{array}{cc}
0.1 & 0.1 \\
0 & 0.1
\end{array}\right] \text {, } \\
& W(1)=\left[\begin{array}{cccc}
-7 & 3 & 2 & 2 \\
3 & -8 & 2 & 3 \\
2 & 2 & -6 & 2 \\
2 & 3 & 2 & -7
\end{array}\right] \text {, } \\
& W(2)=\left[\begin{array}{cccc}
-7 & 2 & 3 & 2 \\
2 & -6 & 2 & 2 \\
3 & 2 & -7 & 2 \\
2 & 2 & 2 & -6
\end{array}\right] \text {, } \\
& W(3)=\left[\begin{array}{cccc}
-8 & 3 & 3 & 2 \\
3 & -7 & 2 & 2 \\
3 & 2 & -7 & 2 \\
2 & 2 & 2 & -6
\end{array}\right] \text {, } \\
& \Gamma(1)=\operatorname{diag}\{4,3\}, \Gamma(2)=\operatorname{diag}\{3,3\} \text {, } \\
& \Gamma(3)=\operatorname{diag}\{4,4\}, \tau_{1,1}=2, \tau_{2,1}=7 \text {, } \\
& \tau_{3,1}=1.2, \quad \tau_{1,2}=1, \tau_{2,2}=6, \tau_{3,2}=1 \text {, } \\
& \tau_{1,3}=3, \tau_{2,3}=6.5, \tau_{3,3}=0.8 \text {. }
\end{aligned}
$$

The activation functions are taken as follows

$$
\begin{aligned}
& f_{1}(s)=g_{1}(s)=h_{1}(s)=\tanh (-0.6 s), \\
& f_{2}(s)=g_{2}(s)=h_{2}(s)=0.4 \tanh (s)
\end{aligned}
$$

and the delayed kernel function is given by $\varphi(s)=\mathrm{e}^{-3 s}$. It is not difficult to verify that

$$
\begin{aligned}
& L_{1}=\Sigma_{1}=\Upsilon_{1}=\operatorname{diag}\{0,0\}, \\
& L_{2}=\Sigma_{2}=\Upsilon_{2}=\operatorname{diag}\{-0.3,0.2\}
\end{aligned}
$$

With the parameters given above and by using the Matlab LMI toolbox, we solve the LMI (11) and obtain the following feasible solutions:

$$
\begin{aligned}
& P_{1}=\left[\begin{array}{cc}
0.7524 & -0.0691 \\
-0.0691 & 1.0418
\end{array}\right], P_{2}=\left[\begin{array}{cc}
1.3868 & -0.1239 \\
-0.1239 & 1.3968
\end{array}\right] \text {, } \\
& P_{3}=\left[\begin{array}{cc}
0.7541 & -0.0940 \\
-0.0940 & 1.2698
\end{array}\right], Q=\left[\begin{array}{cc}
0.0020 & -0.0004 \\
-0.0004 & 0.0037
\end{array}\right] \text {, } \\
& R=\left[\begin{array}{ll}
2.1168 & 0.0213 \\
0.0213 & 1.5872
\end{array}\right], \quad S=\left[\begin{array}{cc}
0.1123 & -0.0000 \\
-0.0000 & 0.1123
\end{array}\right] \text {, } \\
& \Lambda_{1}=\operatorname{diag}\{6.4715,5.9952\}, \quad \Theta_{1}=\operatorname{diag}\{21.9896,18.3192\} \text {, } \\
& \Omega_{1}=\operatorname{diag}\{7.0265,6.7963\}, \Lambda_{2}=\operatorname{diag}\{7.4679,7.5336\} \text {, } \\
& \Theta_{2}=\operatorname{diag}\{22.4617,18.5736\}, \quad \Omega_{2}=\operatorname{diag}\{6.8619,6.5830\} \text {, } \\
& \Lambda_{3}=\operatorname{diag}\{7.0148,6.7889\}, \quad \Theta_{3}=\operatorname{diag}\{21.9830,18.3069\}, \\
& \Omega_{3}=\operatorname{diag}\{7.0304,6.8070\} \text {. }
\end{aligned}
$$

Therefore, it follows from Theorem 1 that the system (8) with given parameters is globally asymptotically synchronized in mean square. The numerical simulation further confirms the theoretical results. Fig. 1 and Fig. 2 display the evolution of the states of the first neutral-type neural network without coupling and with coupling, respectively. Fig. 3 shows that the synchronization error $\operatorname{err}(t)$ approaches zero as $t \rightarrow \infty$.

Remark 5: It is worth to pointing out that the example given above is non-trivial. Note that the system matrix $A(r(t))$ of the single network is unstable, which results in the instability of either single network or coupled system. This can also be observed from Fig. 1 and Fig. 2. Nevertheless, as shown in Fig. 3, the coupled system is synchronized. The numerical simulation is in complete accord with the theoretical results.

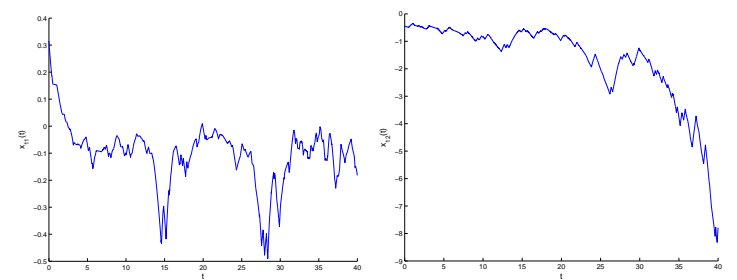

Fig. 1. State Evolution of Single Network Without Coupling.

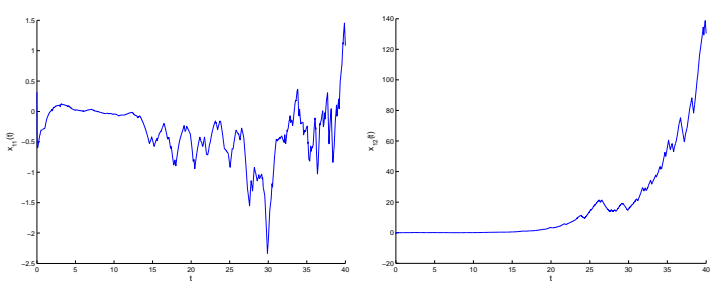

Fig. 2. State Evolution of Single Network in Coupled System. 


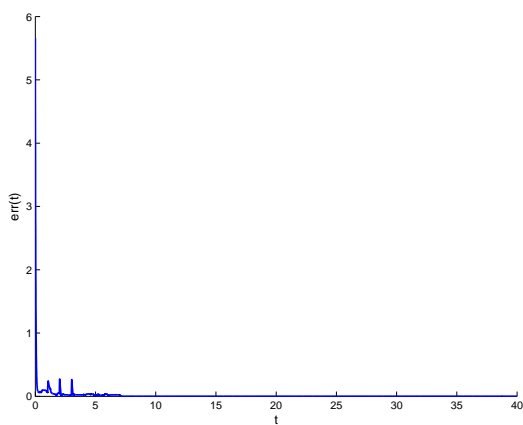

Fig. 3. State Trajectory of the Synchronization Error.

\section{CONCLUSiOnS}

In this paper, we have investigated the synchronization problem for an array of linearly coupled neutral-type neural networks with Markovian jumping parameters and mixed time delays. The discrete time delays are mode-dependent, and distributed time delay is unbounded with mode-dependent upper bound. By utilizing a novel Lyapunov-Krasovskii functional and the Kronecker product, we have shown that the addressed synchronization problem is solvable if several linear matrix inequalities (LMIs) are feasible. A unified LMI approach has been developed to establish sufficient conditions for the coupled neural networks to be globally synchronized in mean square. A numerical example has been provided to show the usefulness of the proposed global synchronization condition.

\section{REFERENCES}

[1] S. Arik, Stability analysis of delayed neural networks, IEEE Transactions on Circuits Systems-I, vol. 47, pp. 1089-1092, 2000.

[2] P. Balasubramaniam, G. Nagamani and R. Rakkiyappan, Passivity analysis for neural networks of neutral type with Markovian jumping parameters and time delay in the leakage term, Communications in Nonlinear Science and Numerical Simulation, vol. 16, no. 11, pp. 44224437, 2011.

[3] H. Bao and J. Cao, Stochastic global exponential stability for neutraltype impulsive neural networks with mixed time-delays and Markovian jumping parameters, Communications in Nonlinear Science and Numerical Simulation, vol. 16, no. 9, pp. 3786-3791, 2011.

[4] A. Bellen, N. Guglielmi and A. Ruechli, Methods for linear systems of circuit delay diferential equations of neutral type, IEEE Transactions on Circuits and Systems-I: Fundamental Theory and Applications, vol. 46, no. 1, pp. 212-216, 1999.

[5] Y. Bengio, P. Frasconi and P. Simard, The problem of learning long-term dependencies in recurrent networks, In Proc. 1993 IEEE Int. Conf. Neural Networks, vol. 3, pp. 1183-1188, 1993.

[6] S. Boyd, L. E. Ghaoui, E. Feron and V. Balakrishnan, Linear matrix inequalities in system and control theory, Philadephia: SIAM, 1994

[7] J. Cao and J. Lu, Adaptive synchronization of neural networks with or without time-varying delays, Chaos, vol. 16, pp. 013133-1-013133-6, 2006.

[8] C. Cheng, T. Liao, J. Yan and C. Hwang, Globally asymptotic stability of a class of neutral-type neural networks with delays, IEEE Trans. Systems, Man and Cybernetics - Part B, vol. 36, no. 5, pp. 1191-1195, 2006.

[9] L. Cheng, Z. Hou and M. Tan, A neutral-type delayed projection neural network for solving nonlinear variational inequalities, IEEE Trans. Circuits and Systems II - Express Brief, vol. 55, no. 8, pp. 806810, 2008.

[10] L. Crocco, Aspects of combustion stability in liquid propellant rocket motors, part I: Fundamentals-low frequency instability with monopropellants, Journal of the American Rocket Society, vol. 21, pp. 163-178, 1951.
[11] Y. Fiagbedzi and A. Pearson, A multistage reduction technique for feedback stabilizing distributed time-lag systems, Automatica, vol. 23, pp. 311-326, 1987.

[12] K. Gopalsamy and X. Z. He, Delay-independent stability in bidirectional associative memory networks, IEEE Trans. Neural Networks, vol. 5, pp. 998-1002, 1994.

[13] W. He and J. Cao, Exponential synchronization of hybrid coupled networks with delayed coupling, IEEE Trans. Neural Networks, vol. 21, no. 4, pp. 571-583, 2010

[14] J. Jost and M. Joy, Special properties and synchronization in coupled map lattices, Phys. Rev. E, vol. 65, 061201, 2002.

[15] H. R. Karimi and H. Gao, New delay-dependent exponential $H_{\infty}$ synchronization for uncertain neural networks with mixed time delays, IEEE Trans. Systems, Man and Cybernetics - Part B, vol. 40, no. 1 , pp. 173-185, 2010.

[16] H. Li, H. Gao and P. Shi, New passivity analysis for neural networks with discrete and distributed delays, IEEE Trans. Neural Networks, vol. 21, no. 11 , pp. 1842-1847, 2010 .

[17] X. Li, H. Gao and X. Yu, A unified approach to the stability of generalized static neural networks with linear fractional uncertainties and delays, IEEE Trans. Systems, Man and Cybernetics - Part B, vol. 41, no. 5 , pp. 1275-1286, 2011.

[18] J. Liang, J. Cao and D. W. C. Ho, Discrete-time bidirectional associative memory neural networks with variable delays, Physics Letters A, vol. 335 , no. 2-3, pp. 226-234, 2005

[19] C. Lien, K. Yu, Y. Lin, Y. Chung and L. Chung, Global exponential stability for uncertain delayed neural networks of neutral type with mixed time delays, IEEE Trans. Systems, Man and Cybernetics - Part $B$, vol. 38, no. 3, pp. 709-720, 2008.

[20] Y. Liu, Z. Wang, J. Liang and X. Liu, Stability and synchronization of discrete-time Markovian jumping neural networks with mixed modedependent time-delays, IEEE Transactions on Neural Networks, vol. 20 no. 7, pp. 1102-1116, 2009

[21] Y. Liu, Z. Wang and X. Liu, Global exponential stability of generalized recurrent neural networks with discrete and distributed delays, Neural Networks, vol. 19, no. 5, pp. 667-675, 2006.

[22] Y. Liu, Z. Wang and X. Liu, On synchronization of coupled neural networks with discrete and unbounded distributed delays, Int. J. Computer Mathematics, vol. 85, no. 8, pp. 1299-1313, 2008.

[23] J. Lu, D. W. C. Ho, J. Cao and J. Kurths, Exponential synchronization of linearly coupled neural networks with impulsive disturbances, IEEE Trans. Neural Networks, vol. 22, no. 2, pp. 329-335, 2011.

[24] W. Lu, L. Wang and T. Chen, On attracting basins of multiple equilibria of a class of cellular neural networks, IEEE Trans. Neural Networks, vol. 22, no. 3, pp. 381-394, 2011.

[25] Y. Niu, J. Lam and X. Wang, Sliding-mode control for uncertain neutral delay systems, IEE Proc. Part D: Control Theory Appl., Vol. 151, No. 1, pp. 38-44, 2004.

[26] J. Park, Synchronization of cellular neural networks of neutral type via dynamic feedback controller, Chaos Solitons and Fractals, vol. 42, no. 3 , pp. 768-778, 2009.

[27] L. M. Pecora and T. L. Carroll, Synchronization in chaotic systems, Phys. Rev. Lett., vol. 64, no. 8, pp. 821-824, 1990.

[28] V. Perez-Munuzuri, V. Perez-Villar, L. O. Chua, Autowaves for image processing on a two-dimensional CNN array of excitable nonlinear circuits: flat and Wrinkled labyrinths, IEEE Trans. Circuits Syst. -I, vol. 40, pp. 174-181, Mar. 1993

[29] J. C. Principle, J. M. Kuo, and S. Celebi, An analysis of the gamma memory in dynamic neural networks, IEEE Trans. Neural Networks, vol. 5, no. 2, pp. 337-361, 1994.

[30] H. Qiao, J. Peng and Z. B. Xu, A reference model approach to stability analysis of neural networks, IEEE Trans. Systems, Man and Cybernetics - Part B, vol. 33, no. 6, pp. 925-936, 2003.

[31] Y. Shen and J. Wang, Noise-induced stabilization of the recurrent neural networks with mixed time-varying delays and Markovian-switching parameters, IEEE Trans. Neural Networks, vol. 18, no. 6, 1857-1862, 2007.

[32] J. E. Slotine, W. Li, Applied Nonlinear Control, Prentice-Hall, New Jersey, 1991.

[33] P. Tino, M. Cernansky, and L. Benuskova, Markovian architectural bias of recurrent neural networks, IEEE Trans. Neural Networks, vol. 15, no. 1, pp. 6-15, 2004.

[34] X. Wang and G. Chen, Synchronization in small-world dynamical networks, Int. J. Bifurc. Chaos, vol. 12, no. 1, pp. 187-192, 2002.

[35] Z. Wang, Y. Liu, M. Li and X. Liu, Stability analysis for stochastic Cohen-Grossberg neural networks with mixed time delays, IEEE Transactions on Neural Networks, vol. 17, no. 3, pp. 814-820, 2006. 
[36] Z. Wang, Y. Liu, L. Yu and X. Liu, Exponential stability of delayed recurrent neural networks with Markovian jumping parameters, Physics Letters A, vol. 356, no. 4-5, pp. 346-352, 2006.

[37] C. W. Wu, Synchronization in arrays of coupled nonlinear systems with delay and nonreciprocal time-varying coupling, IEEE Trans. Circuits Syst. $-I I$, vol. 52, no. 5, pp. 282-286, 2005.

[38] Z. Wu, P. Shi, H. Su and J. Chu, Passivity analysis for discrete-time stochastic Markovian jump neural networks with mixed time delays, IEEE Trans. Neural Networks, vol. 22, no. 10, pp. 1566-1575, 2011.

[39] R. Yang, Z. Zhang and P. Shi, Exponential stability on stochastic neural networks with discrete interval and distributed delays, IEEE Trans. Neural Networks, vol. 21, no. 1, pp. 169-175, 2010.

[40] Y. Zhang, S. Xu, Y. Chu and J. Lu Robust global synchronization of complex networks with neutral-type delayed nodes, Applied Mathematics and Computation, vol. 216, no. 3, pp. 768-778, 2010.

[41] A. Zheleznyak, L. O. Chua, Coexistence of low- and high-dimensional spatio-temporal chaos in a chain of dissipatively coupled Chua's circuits, Int. J. Bifurcation and Chaos, vol. 4, no. 3, pp. 639-674, 1994.

[42] Q. Zhu and J. Cao, Exponential stability of stochastic neural networks with both Markovian jump parameters and mixed time delays, IEEE Trans. Systems, Man and Cybernetics - Part B, vol. 41, no. 2, pp. 341353, 2011.

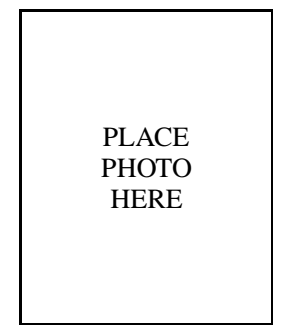

ics.
Yurong Liu received his B.Sc. degree in Mathematics from Suzhou University, Suzhou, China, in 1986, the M.Sc. degree in Applied Mathematics from Nanjing University of Science and Technology, Nanjing, China, in 1989, and the Ph.D. degree in Applied Mathematics from Suzhou University, Suzhou, China, in 2000.

Dr. Liu is now a Professor at the Department of Mathematics, Yangzhou University, China. His current interests include neural networks, nonlinear dynamics, time-delay systems, and chaotic dynam-

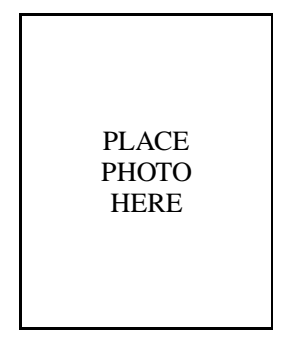

Zidong Wang was born in Jiangsu, China, in 1966. $\mathrm{He}$ received his B.Sc. degree in Mathematics in 1986 from Suzhou University, Suzhou, China, the M.Sc. degree in Applied Mathematics in 1990 and the Ph.D. degree in Electrical and Computer Engineering in 1994, both from Nanjing University of Science and Technology, Nanjing, China.

Currently, he is a Professor of Dynamical Systems and Computing at Brunel University in the UK. His research interests include dynamical systems, signal processing, bioinformatics, control theory and applications. He has published more than 200 papers in refereed international journals.

Prof. Wang is currently serving as an Associate Editor for 12 international journals including IEEE Transactions on Automatic Control, IEEE Transactions on Neural Networks, IEEE Transactions on Signal Processing, IEEE Transactions on Systems, Man, and Cybernetics - Part C and IEEE Transactions on Control Systems Technology.

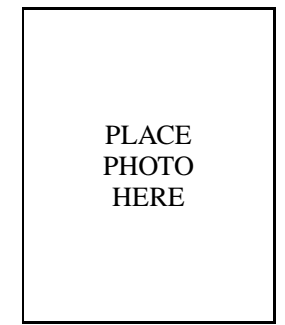

Jinling Liang received the B.Sc. and M.Sc. degrees in mathematics from Northwest University, $\mathrm{Xi}$ ' an, China, in 1997 and 1999, respectively, and the $\mathrm{Ph} . \mathrm{D}$. degree in applied mathematics from Southeast University, Nanjing, China, in 2006.

Currently, she is a Professor at the Department of Mathematics, Southeast University. From January 2004 to March 2004, she was a Research Assistant at the University of Hong Kong. From March to April 2004, she was a Research Assistant at the City University of Hong Kong. From April 2007 to March 2008, she was a Postdoctoral Research Fellow at the Department of Information Systems and Computing, Brunel University, Uxbridge, Middlesex, U.K., sponsored by the Royal Society Sino-British Fellowship Trust Award of the United Kingdom. She has published around 20 papers in refereed international journals. Her research interests include neural networks, complex networks, nonlinear systems, and bioinformatics.

Dr. Liang is an Associate Editor of Neurocomputing, a member of program committee for some international conferences, and a very active reviewer for many international journals.

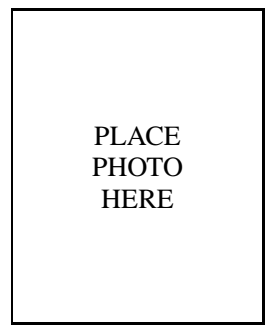

Xiaohui Liu is Professor of Computing at Brunel University in the UK where he directs the Centre for Intelligent Data Analysis, conducting interdisciplinary research concerned with the effective analysis of data. He was Honorary Pascal Professor at Leiden University (2004) and Visiting Researcher at Harvard Medical School (2005). Professor Liu is a Charted Engineer, Life Member of the Association for the Advancement of Artificial Intelligence, Fellow of the Royal Statistical Society, and Fellow of the British Computer Society. He has given numerous invited and keynote talks, chaired several international conferences, and advised funding agencies on interdisciplinary research programmes. Collaborating with many talented physical, clinical and life scientists, Professor Liu has over 250 publications in biomedical informatics, data mining, dynamic and intelligent systems. 